\title{
Numerical Study of Gas-liquid Micro-cyclone Separator Flow Field
}

\author{
Peng Qian \\ State-key Laboratory of Chemical Engineering \\ East China University of Science and Technology \\ Shanghai, China

\section{Liang Ma} \\ State-key Laboratory of Chemical Engineering \\ East China University of Science and Technology \\ Shanghai, China
}

\author{
Yi Liu \\ State-key Laboratory of Chemical Engineering \\ East China University of Science and Technology \\ Shanghai, China \\ Yanhong Zhang* \\ State-key Laboratory of Chemical Engineering \\ East China University of Science and Technology \\ Shanghai, China \\ e-mail: zhyh75@ecust.edu.cn
}

\begin{abstract}
Based on Reynolds stress model (RSM), the threedimensional flow field of a gas-liquid micro-cyclone separator with a diameter of $75 \mathrm{~mm}$ have been simulated with the computational fluid dynamics (CFD). The distribution of gas tangential velocity of the inside flow field and its effects on the movement of liquid droplets are presented in this paper. The results suggest that the micro-cyclone accords with the vortex motion rules and the droplets fractional efficiency change with the inlet flow rate. Also, an efficient separation zone of the micro-cyclone have been found.
\end{abstract}

Keywords- CFD; Micro-cyclone; Numerical study; Tangential velocity

\section{INTRODUCTION}

The gas-liquid micro-cyclone is used to implement gasliquid separation by centrifugal force with a simple structure and without moving parts. The 3D turbulent flow of microcyclone is a very complicated. To investigate the flow characteristics, the mechanism of separation and set up mathematical models of the internal flow field, theoretical analysis, tests and numerical simulation were generally used. In recent years, the micro-cyclone had undertaken extensive research by CFD simulation [1-15], because the research of theoretical analysis and tests hasn't applied to the separation of complex turbulent flow. Recent studies show that RSM turbulent models are in very good agreement with the measured value by used in simulating the mean velocity and static pressure.

In this article, CFD simulation technology was used to simulate the flow field inside the gas-liquid micro-cyclone separator with a diameter of $75 \mathrm{~mm}$. Simulation with established models shows that the flow field and tangential velocity inside the micro-cyclone separator were changed. Similarly, the areas with different tangential velocities changed under different processing volumes.

\section{EXPERIMENTAL}

\section{A. Computing Models}

1) The mass and momentum conservation equations of incompressible fluid at mean-time Reynolds number:

$$
\begin{gathered}
\frac{\partial u_{i}}{\partial x_{i}}=0 \\
\frac{\partial u_{i}}{\partial t}+\frac{\partial}{\partial x_{j}}\left(u_{i} u_{j}\right)=\frac{\partial p}{\partial x_{i}}+v \frac{\partial}{\partial x_{j}}\left(\frac{\partial u_{i}}{\partial x_{j}}+\frac{\partial u_{j}}{\partial x_{i}}\right)-\frac{\partial \tau_{i j}}{\partial x_{j}}+f_{i} \\
\tau_{i j}=\mu_{t}\left(\frac{\partial u_{j}}{\partial x_{i}}+\frac{\partial u_{i}}{\partial x_{j}}\right)-\frac{2}{3} \mu_{t} \delta_{i j} \frac{\partial u_{j}}{\partial x_{j}}
\end{gathered}
$$

2) The transport equation of the RSM model:

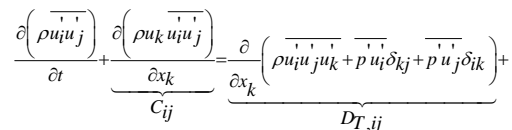

$$
\begin{aligned}
& \underbrace{\frac{\partial}{\partial x_{k}}\left[\mu \frac{\partial}{\partial x_{k}}\left(\mu_{i}^{\prime} \mu_{j}^{\prime}\right)\right]}_{D_{L, i j}}-\underbrace{-\left(\overline{\mu_{i}^{\prime} \mu_{k}^{\prime}} \frac{\partial u_{j}}{\partial x_{k}}+\overline{\mu_{j}^{\prime} \mu_{k}^{\prime}} \frac{\partial u_{i}}{\partial x_{k}}\right)}_{P_{i j}} \underbrace{\rho \beta\left(\overline{\left.g_{i} \overline{u_{j}^{\prime} \theta}+g_{j} \overline{u_{i}^{\prime} \theta}\right)}\right.}_{G_{i j}} \\
& \underbrace{p^{\prime}\left(\frac{\partial u_{i}^{\prime}}{\partial x_{j}}+\frac{\partial u_{j}^{\prime}}{\partial x_{i}}\right)}_{\varphi_{i j}} \underbrace{-2 \mu \frac{\partial u_{i}^{\prime} u_{j}^{\prime}}{\partial x_{k} \partial x_{k}}}_{\varepsilon_{i j}} \underbrace{2 \rho \Omega_{k}\left(\overline{u_{j}^{\prime} u_{m}^{\prime}} e_{i k m}+\overline{u_{i}^{\prime} u_{m}^{\prime}} e_{j k m}\right)}_{F_{i j}}
\end{aligned}
$$

In the formula, the first term is transient phase, $\mathrm{C}_{\mathrm{ij}}$ is convection phase, $\mathrm{D}_{\mathrm{T} . \mathrm{j} \text { is }}$ turbulent flow diffusion phase, $\mathrm{D}_{\mathrm{L} . \mathrm{ij}}$ is molecular viscosity diffusion phase, $\mathrm{P}_{\mathrm{ij}}$ is shear stresses part, $G_{i j}$ is buoyancy part, ij is pressure strain term, $\varepsilon_{i j}$ is viscous dissipation, $\mathrm{F}_{\mathrm{ij}}$ is system rotates

\section{B. Geometrical Model and Mesh Dividing}

The cyclone separator have been studied by many researchers with different structures and sizes [16-18], but it is rare for the micro-cyclone separator with the cylindrical section diameter of $75 \mathrm{~mm}$. As shown in Figure 1, the structure dimension of the micro-cyclone separator involves: diameter of the cylindrical section is $75 \mathrm{~mm}$, and ordinate is origin, and the origin of coordinates in the central axis of the top cover of micro-cyclone separator, positive direction is down. 
Owing to the sharp degree of connection pipe and cylinder, take method of block grid generation. In different regions were using a structured grid, grid node number is 370000 .

\section{The Mathematical Model and Boundary Conditions}

Based on the Reynolds stress model (RSM), unsteady incompressible turbulent flow in the micro-cyclone separator under different flow was simulated by establishing the discrete equations and using QUICK difference scheme and SIMPLE algorithm for solving the control equation. In addition, the motion trajectory of droplets was simulates using a discrete phase model (DPM).
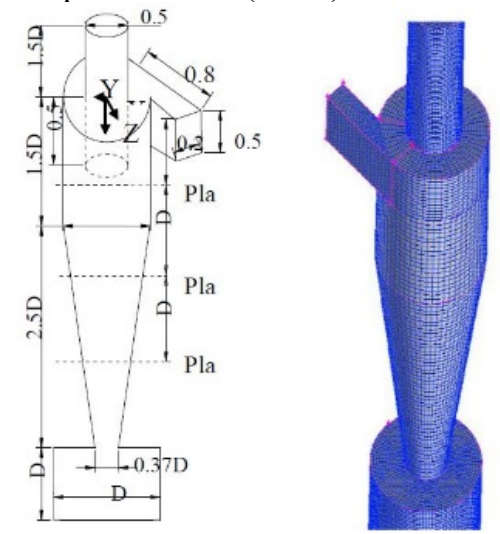

Figure 1. (a) Geometric figure of the micro-cyclone separator, (b) Grids layout of the micro-cyclone separator.

a) Inlet boundary conditions: inlet flow is normal temperature air, Entrance velocity is $4 \mathrm{~m} / \mathrm{s}, 8 \mathrm{~m} / \mathrm{s}, 12 \mathrm{~m} / \mathrm{s}$, $16 \mathrm{~m} / \mathrm{s}$. The inlet boundary condition for DPM is the Escape boundary conditions. In other words, the droplets return to the surface to break away from the boundaries. Thus, the computational domains were not to be calculated.

b) Outlet boundary conditions: according to the fully developed tube flow conditions, the gradient of all variables is 0 in normal of the exit section. DPM export settings is escape (Escape) boundary conditions. When the droplet reaches the surface, stop tracking the droplet. Track results are marked as "Escape".

c) Wall condition: using nonslip velocity condition. DPM wall settings is trap boundary conditions. This is, once the droplet collision the wall, the droplet is trapped and termination of orbit calculation of the droplets.

\section{RESULTS AND DISCUSSION}

\section{A. Flow Field Distribution}

It can be concluded that tangential velocity plays an important role in the three dimensional gas-liquid movement of the micro-cyclone separator because of the faster speed moving than axial velocity and the imperative condition of producing centrifugal force. Therefore, tangential velocity is the most important performance metric for micro-cyclone separator.

The tangential is consistent basically with the combination vortex movement. The radius of micro-cyclone separator is divided into three vortex regions: forced vortex region, quasi-free vortex region and boundary layer. The region that radius is from 0 to half-radius of exit tube is the forced vortex region with a linear distribution. And the tangential velocity intensifies sharply and maximum value is approximately twice than the inlet. The next region, to the boundary layer, is the quasi-free vortex region, which tangential velocity is slowly decreasing. In the figures we can deduce the variation tendency of tangential velocity is basically the same.

\section{B. Droplets Fractional Efficiency}

According to the simulation of the discrete phase, the droplets fractional efficiency can be obtained by

$$
\eta=\frac{\text { The number of particles trapped }}{\text { The number of particles released }} \times 100 \%
$$
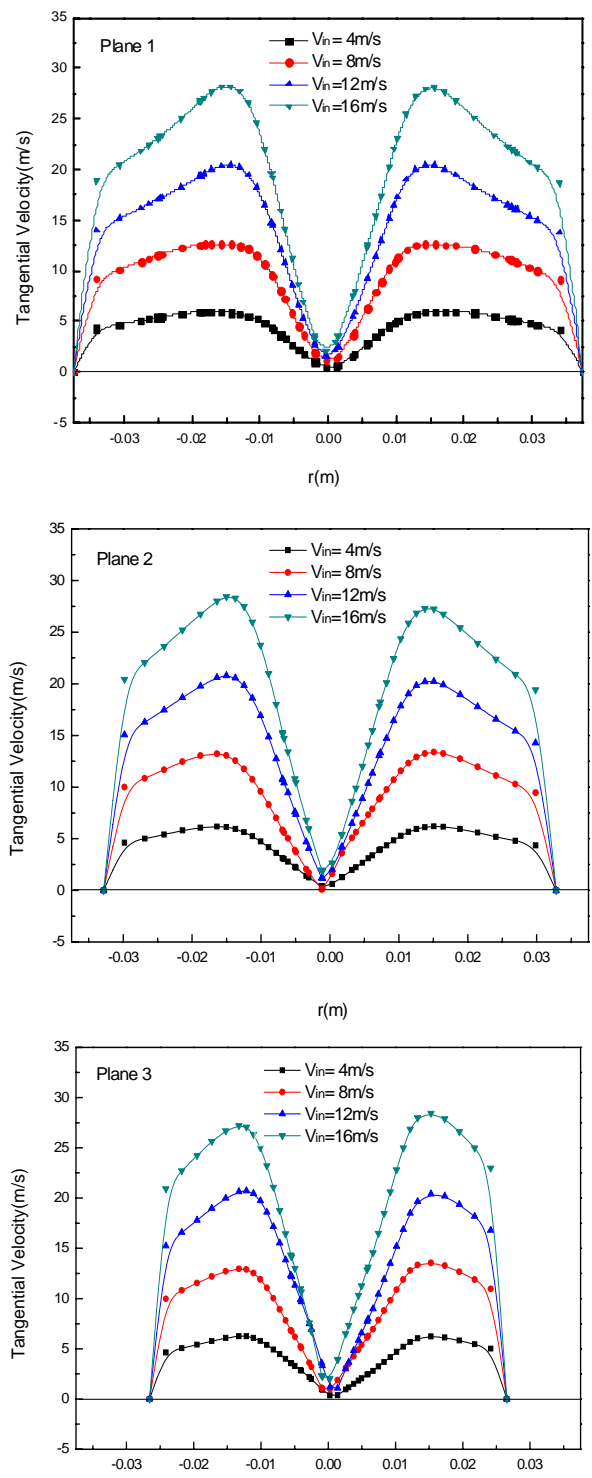

$r(m)$

Figure 2. Tangential velocity profiles with different flow rates. 
In this study, the separation properties of four different flow rates were simulated under droplets diameters of 0.5 to $10 \mu \mathrm{m}$, as Figure 3 shows. It shows how different flow rates impact the droplets fractional efficiency. It is clearly observed that the droplets capture efficiency of microcyclone separator rises with the increase of droplets diameter. The droplets diameter greater than $7 \mu \mathrm{m}$ were almost completely captured. For the $75 \mathrm{~mm}$ micro-cyclone separator, when the flow rates was $12 \mathrm{~m} / \mathrm{s}$, the droplets fractional efficiency is highest, and the maximum efficiency is close to $100 \%$. And when the flow rates was $8 \mathrm{~m} / \mathrm{s}$ or $16 \mathrm{~m} / \mathrm{s}$, the efficiency remains high. Based on this simulation data, if the flow rate is low, it's not enough to generate the centrifugal force to separate the droplets from gas out the micro-cyclone separator. The alternative to this is excessive flow rate, the settle time of droplets in micro-cyclone separator is too short to be captured. In such conditions, it adds the short-circuit flow and increases the gas turbulence, and thus the droplets are brought again. Thus, it shows that if the flow rate is too large or small, the droplets capture efficiency is greatly reduced.

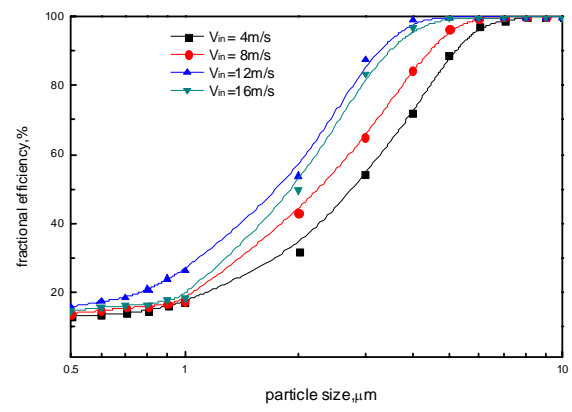

Figure 2. Droplets fractional efficiency with different flow rates

\section{CONCLUSION}

In this paper, the numerical simulation of the flow distribution in micro-cyclone separator with RSM model was performed by using CFD. And the droplets fractional efficiency of micro-cyclone separator was calculated using DPM model.

The following conclusions were reached: the tangential degree in micro-cyclone separator basically line with the combination vortex motion, from the axis to the wall, the tangential velocity first increases rapidly to nearly twice of the inlet flow rate, then decreased slowly, and when arrive in the boundary layer region, it dropped sharply to 0 . For the $75 \mathrm{~mm}$ micro-cyclone separator, the droplets diameter greater than $7 \mu \mathrm{m}$ were almost completely captured. And there is an effective separation area within the flow rates range with $8 \mathrm{~m} / \mathrm{s}$ to $16 \mathrm{~m} / \mathrm{s}$.

\section{REFERENCES}

[1] Boysan F, Ayers W H, withenbank J,"A fundamental mathematical modeling approach to cyclone design,"Transactions of the Institution of Chemical Engineers, 1982, 60: 222 230.

[2] Ovadia, "State of the art of gas-liquid cylindrical-cyclone conpactorseparators technology," JPT July 1998.
[3] Derksen J J, "Separation performance predictions of a stairmand highefficiency cyclone," AIChE J, 2003, 49 (6): 1359 1370.

[4] Hu L Y, Zhou L X, Shi M X, "Studies on strongly swirling flows in the full space of a volute cyclone separator," AIChE J, 2005, 51 (3): 740 749.

[5] Shalaby H, Pachler K, Wozniak K, "Comparative study of the continuous phase flow in a cyclone separator using different mrbulence models," International Journal for Numerical Methods in Fluids, 2005, 48: 1175 1197.

[6] Narasimha M, Sripriya, Banerjee P K, "CFD modelling of hydrocyclone-prediction of cut size," International Journal of Mineral Processing, 2005 (75): 53 68.

[7] Narasimha $M$, Brennan $M$, Holtham $P$ N, "Large eddy simulmion ofhydr cyclone- prediction of air-core diameter and shape," International Journal ofMineral Processing, 2006 (80): 1 14.

[8] Murphy S, Delfos R, Pourqui6b M J B M, "Prediction of strongly swirling flow within an axial hydrocyclone usingtwo commercial CFD codes," Chemical Engineering Science, 2007 (62): 1619 1635.

[9] Bhaskar K U, Murthy Y R, Raju M R, "CFD simulmion and experimental validation studies on hydrocyclone."Minerals Engineering, 2007 (20): 60 71.

[10] Martignoni W P, Bemardo S, Quintani C L, "Evaluation of cyclone geometry and its influence on performance parameters by computational fluid," Brazilian Journal of Chemical Engineering,2007, 24 (1): 83 94.

[11]Derksen J J, Van den Akker H E A,"Simdmion of Vortex Core Precession in a Reverse-Flow Cyclone,"AIChE Journal, 2000, 46 (7): 1317 1331.

[12] Gimbun J, Chuah T G, “The influence of temperature and inlet velocity on cyclone pressure drop: a CFD study,"Chemical Engineering and Processing, 2005 (44): 7 12.

[13] Yoshida H, Norimoto U, Fukui K, "Effect of blade rotation on particle classification performance of hydro-cyclones,"Powder Technology, 2006 (164): 103 110

[14]Lee J W, Yang H J, Lee D Y,"Effect of the cylinder shape of a longconed cyclone on the stable flow-field establishment,"Powder Technology 2006(165): 30 38.

[15] Saengchana K, Nopharatanab A, Songkasiri W,"Enhancement of tapioca starch separation with a hydrocyclone: effects of apex diameter, feed concentration, andpressure drop on tapioca starch separation with a hydrocyclone,"Chemical Engineering and Processing, 2009 (48): 195 202.

[16] J.K. Briant, O.R. Moss, "The influence of electrostatic charge on the performance of 10-mm nylon cyclones,"American Industrial Hygiene Association Journal, 1984, 45(7): 440-445.

[17] KoubaGE, Shoham O, ShiraziS, "Design and performance of gas-liquid cylindrical cyclone separators,"BHR Group 7th International Conference on Multiphase Flow, 1995: 307-327.

[18] Alexander RM,"Fundamentals of cyclone design and operation. Proceedings of the Australian Institute of Mining Metals,"1949, 152153: 203-228 